\title{
Airway Management Considerations for Upper Gastrointestinal Endoscopic Procedures in COVID-19 Era
}

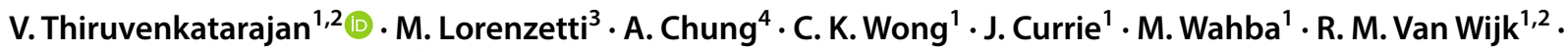 \\ M. W. Skinner ${ }^{5} \cdot$ M. Sorbello ${ }^{6}$
}

Received: 4 June 2020 / Accepted: 4 July 2020 / Published online: 25 July 2020

(c) Springer Science+Business Media, LLC, part of Springer Nature 2020

Keywords Endoscopy $\cdot$ COVID-19 $\cdot$ Anesthesia $\cdot$ Airway management $\cdot$ SAD

Abbreviations

COVID-19 Coronavirus disease 2019

SARS-CoV-2 Severe acute respiratory syndrome coronavirus 2

AGP Aerosol generating procedures

PCR Polymerase chain reaction

PPE Personal protective equipment

ACG American college of gastroenterology

DHPA Digestive health physicians association

ERCP Endoscopic retrograde cholangiopancreatography

HFNC High flow nasal cannula

LMA Laryngeal mask airway

SAD Supraglottic airway device
To the Editors,

We read with interest the paper by Lauro and colleagues highlighting the emergency endoscopy experience during the SARS-CoV-2 pandemic in the north of Italy [1]. Aerosol and droplet generation during upper gastrointestinal endoscopies is attributed to scope insertion into the pharynx, instrument manipulation through the endoscope channel and positive gas insufflation, that is often required to facilitate esophageal intubation with the endoscope. [2, 3]. Aerosol and droplet generation can be further augmented by airway manipulations and lighter planes of anesthesia resulting in patients bucking or coughing. The exposure to infection for anesthetists and endoscopists is high due to their proximity to the airway [4]. We outline airway management principles for endoscopy in known/suspected as well as unsuspected COVID-19 patients, based on existing guidelines and our practice adaptations.
V. Thiruvenkatarajan

venkatesan.thiruvenkatarajan@sa.gov.au

M. Lorenzetti

marklorenzetti@internode.on.net

A. Chung

chung_adrian@hotmail.com

C. K. Wong

chunkeat.wong@gmail.com

J. Currie

john.currie@sa.gov.au

M. Wahba

medhat.wahba@sa.gov.au

R. M. Van Wijk

roelof.vanwijk@sa.gov.au

M. W. Skinner

marcus.skinner@ths.tas.gov.au
M. Sorbello

maxsorbello@gmail.com

1 Department of Anesthesia, The Queen Elizabeth Hospital, Woodville, Adelaide, SA 5011, Australia

2 Discipline of Acute Care Medicine, University of Adelaide, Adelaide, Australia

3 Department of Gastroenterology, The Queen Elizabeth Hospital, Adelaide, Australia

4 Department of Gastroenterology and Hepatology, Flinders Medical Centre, Adelaide, Australia

5 Surgical and Perioperative Services, Royal Hobart Hospital, Hobart, Australia

6 Anaesthesia and Intensive Care, AOU Policlinico San Marco University Hospital, Catania, Italy 
Elective gastrointestinal endoscopies procedure should be cancelled or postponed during the pandemic peak, to limit contagion diffusion [1]. Urgent endoscopies in known or suspected COVID-19 cases should be performed ideally in a negative pressure room with tracheal intubation under appropriate precautions for aerosol generating procedures (AGP) [5]. During the second phase, while recommencing the endoscopy services in United States, there are suggestions that COVID-19 PCR testing should be implemented pre-emptively once the health system is equipped with testing and isolating suspected cases [6]. Notably, the latest AGA/DHPA (American Gastroenterology Association/Digestive Health Physicians Association) statement (April 27, 2020) recommends that all patients should receive PCR-based testing within $48 \mathrm{~h}$ of the procedure whenever possible. If this option is not feasible, a temperature log for 10 days prior to the procedure and a symptom questionnaire and temperature check on the day should be performed [7]. A similar approach is followed at one of the author's (MS) institutions in Italy. It should be, however, noted that the test sensitivity ranges from $70 \%$ to $95 \%$ allowing for false negatives [8]. Locally updated guidelines based on the community transmission risk along with inputs from the infectious disease experts are often the best resources in this regard.

Intubation experiences from Wuhan, China, described applying wet gauze around the nostrils and mouth to limit droplets spread [9]. An adaptation of this approach during endoscopy would involve gently sealing the gap between the mouth guard and the endoscope using a soft wet cloth/gauze. Maintaining a deeper plane of anesthesia, administration of antiemetics and an antisialagogue will reduce droplets generation. Attempting esophageal intubation without gas insufflation (if feasible), shielding the biopsy channel with a soft cloth or air suctioning while withdrawing the instruments, [10] and ceasing the gas flow as the endoscope is withdrawn from the oropharynx are further considerations.

The prevalence rate in the community and inputs from local infectious disease experts should be considered when choosing the best airway management strategy for unsuspected patients. In regions with high prevalence of COVID19, complex interventions such as an endoscopic retrograde cholangiopancreatography (ERCP) are best managed with tracheal intubation. When used with a superimposed surgical mask, High flow nasal cannula (HFNC) may be a reasonable option in hypoxemic patients to avoid intubation [11]. Its use has been described during intubation for critically ill COVID-19 patients, though highly debated [12]. Nonetheless, HFNC therapy assisted sedation is not employed during endoscopy in many centers (anecdotal evidence) regardless of the prevalence rate, due to concerns with aerosolization of viral particles [13]. Further, endoscopy precludes the application of a surgical mask over the HFNC.

Moderate to deep sedation assisted by a standard low flow nasal cannula may be an appropriate approach for simple interventions, and also in urgent cases where the diagnosis is unknown, when tracheal intubation is unwarranted. Models reveal that the viral transmission is feasible through asymptomatic carriers, and the viral loads could be similar between symptomatic and asymptomatic patients [2]. Clearly, the use of a mouth guard and negotiating the endoscope through an open oropharynx during sedation may increase the risk of aerosol and droplet generation in asymptomatic patients. Tracheal intubation can provide a near complete seal of the airway and reduce aerosolization. Yet intubation and emergence phenomenon with associated maneuvers such as suctioning can potentially disperse aerosol and droplets, and tracheal intubation is the most associated route of contagion among airway interventions [14]. An alternate strategy in such instances is to use the LMA ${ }^{\circledR}$ Gastro $^{\mathrm{TM}}$ (Teleflex Medical Europe Ltd, Athlone, Ireland) with a viral filter (paying due attention to ensure a leak-free seal) under general anesthesia. This will suit complex interventions such as an ERCP for biliary obstruction/sepsis in patients without aspiration/difficult airway risk. The device has a high insertion success rate (99\%), maintains oxygenation, has a low (1\%) endoscopy failure rate and has low incidence of serious adverse events [15]. There are no data to suggest that the $\mathrm{LMA}^{\circledR} \mathrm{Gastro}^{\mathrm{TM}}$ technique is safer than routine sedation techniques with an open airway in asymptomatic COVID-19 patients. Nonetheless, due to various qualities of the $\mathrm{LMA}^{\circledR} \mathrm{Gastro}^{\mathrm{TM}}$ it would seem to be a favorable option. First, it is a second generation supraglottic airway device (SAD), hence, it can deliver higher seal pressures during assisted ventilation (when appropriately sized), and may reduce aerosolization of viral particles [16]. Second, it reduces the need for airway manipulation (e.g. chin lift, jaw thrust) and close proximity to the airway. Third, it provides a near complete seal of the oral cavity/oropharynx from its dual channel design as compared to an open airway during sedation (Fig. 1) [15]. Fourth, it negates patient movement and cough (seen with sedation), and facilitates smooth emergence (compared with endotracheal tube). Although SADs placement and removal has been suggested as lower risk AGP [14, 16], we are not aware of any research proving or disproving this. SADs have also been suggested for "closed circuit" intubation in COVID-19 patients to minimize aerosolization [17]. Additional maneuvers to decrease aerosolization include: applying a clear occlusive dressing to seal any gap between the device and the oral cavity; providing an adequate depth of anesthesia to avoid coughing and straining; use of an antisialagogue; consider deep extubation; and, keeping the device attached to the circuit during extubation. The endoscope channel can be sealed with a tape/dressing once the procedure is finished. Limitations include: it is an oral device and is not suitable for patients with limited mouth opening; its dedicated endoscopy channel has a $16 \mathrm{~mm}$ internal diameter and larger endoscopes 


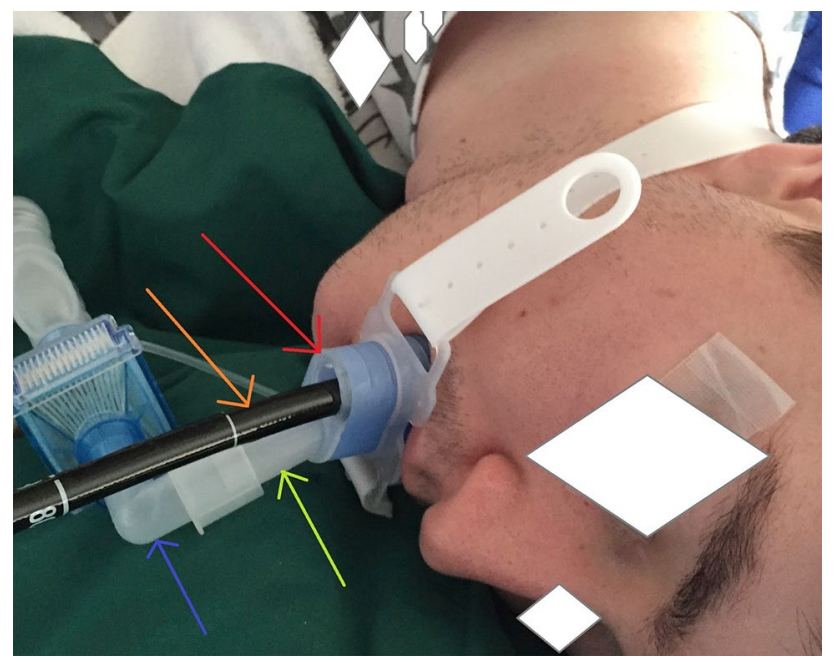

Fig. 1 The $\mathrm{LMA}^{\circledR}$ Gastro ${ }^{\mathrm{TM}}$ airway in place. Breathing circuit with filter (blue arrow), dedicated airway channel (light green arrow), endoscope (orange arrow) inserted through the endoscopy channel (red arrow). [Published with permission from the British Journal of Anaesthesia [12]

( $>14 \mathrm{~mm}$ outer diameter) will not fit; and, patients at high risk of aspiration should have their airway secured with a tracheal tube. Familiarity and device accessibility are further limitations. The authors have been regularly using the device for the last 3 years, and it is evident that an airtight seal is possible by choosing an appropriate size. [15, 18]. In our own experience (MS) during the peak of the pandemic in northern Italy, we found that $\mathrm{LMA}^{\circledR} \mathrm{Gastro}^{\mathrm{TM}}$ was a valuable device. It was used in a scenario where an endoscopy needed to be performed in a highly suspected patient, avoiding tracheal intubation due to concerns of extubation failure post procedure and lack of intensive care beds. The device is used for selected interventions (during anesthesia supported services) at the authors' (VT, MWS, MS) institutions for unsuspected COVID-19 cases. Of note, a recent expert opinion (endorsed by the Difficult Airway Society) on the use of SAD in COVID-19 positive or unknown patients recommend no change in the airway management. The panel suggests that if an SAD is warranted, it should be a second generation device, and a scrupulous attention should be paid to ensuring a leak-free seal [14].

Although any SAD could be used for endoscopy, only few of them are specifically designed for this purpose such as the LMA ${ }^{\circledR} \mathrm{Gastro}^{\mathrm{TM}}$ and the Gastro-laryngeal tube [19]. We believe that these devices might be of benefit especially in COVID unconfirmed or negative patients, and helps in optimal resource utilization of PPE, equipment and medication apart from reducing the healthcare workers exposure. These considerations need to be taken in account either during the outbreak phase, for emergency cases, but also for phase 2 with progressive resumption of elective procedures.

\section{Reply}

\section{Dear Editor,}

We thank Dr. Thiruvenkatarajan and colleagues from the Queen Elizabeth Hospital in Adelaide for their interest in our article entitled: "Emergency Endoscopy During the SARS-CoV-2 Pandemic in the North of Italy: Experience from St. Orsola University Hospital - Bologna" [1]. In their manuscript in press in Digestive Disease and Sciences entitled: "Airway management considerations for upper gastrointestinal endoscopic procedures in COVID19 era", they outline the airway management principles for endoscopy in known/suspected and unsuspected COVID19 patients, based on existing guidelines and their practice adaptations.

During the pre-peak and early peak phase of the SARSCoV-2 pandemic in northern Italy, we performed urgent endoscopies in patients with known or suspected COVID19 infection, reporting no difference when comparing the number of procedures with the same period in 2019.

At the beginning of the pandemic, since a rapid test was not available, we treated every urgent procedure as a potential positive case, using our previously established policy for airway management. In our practice, supported by a recent expert statement [14], supraglottic airway devices bear a similar risk of infection transmission while providing a less secure airway compared with endotracheal tubes; accordingly, we did not change our standard management in patients with suspected or proven COVID-19, adopting orotracheal intubation only when necessary [14] such as proximal to a foreign body or a bolus impaction. In case of upper digestive bleeding, we adopted intubation based on the clinical situation, especially considering the predicted duration and difficulty of the procedure versus the risk of aspiration. When intubation was not required, we performed the procedure during spontaneous breathing, always administering moderate-to-deep sedation, as evidence shows that this approach is safe for most endoscopic procedures [19]. Correctly administered sedation enables the introduction of the endoscope without causing vomiting or coughing, thus reducing droplet generation. Oxygen was supplemented by short nasal cannulae only when necessary, since it increases the risk of aerosol generation. We did not use antiemetic drugs since in our clinical practice they were never needed in this situation.

During the pandemic peak, we performed urgent endoscopies in a negative pressure room, wearing proper personal protective equipment (PPE). For non-emergency endoscopy, a prior negative rapid PCR test was required.

Due to the sustained reduction in infection rate in the late phase of the pandemic, according to the recent international guidelines [7] we resumed elective endoscopic 
activities in outpatients, administering a symptom questionnaire and performing a temperature check on the day of the procedure.

We agree with our colleagues (this article) that some devices could be useful in order to avoid aerosols and droplets but, on the other hand, during the introduction of the device (as in orotracheal intubation), the operator is nevertheless exposed to possible contact with droplets and aerosols, reducing the overall benefit. Compliance with the proper procedures such as donning PPE prior to sedation, with careful doffing afterwards, is the foremost safety precaution aimed at reducing the risk of contagion. No documented transmission of COVID-19 infection to caregivers was attributable to our upper emergency endoscopies, suggesting that our policy, including airway management, was effective in preventing the spread of contagion.

A. Lauro (Corresponding Author), N. Pagano, G. Impellizzeri , M. Cervellera, V. Tonini

Funding The author(s) received no financial support for authorship and/or publication of this article.

\section{Compliance with Ethical Standards}

Conflict of interest MWS is the creator of the $\mathrm{LMA}^{\circledR} \mathrm{Gastro}^{\mathrm{TM}}$ airway. He holds a part-time consultancy agreement with Teleflex. Teleflex did not influence the content of this correspondence nor reviewed this prior to submission. MS has received paid consultancy from Teleflex Medical, Verathon Medical and DEAS Italia, is a patent co-owner (no royalties) of DEAS Italia and has received lecture grants and travel reimbursements from MSD Italia, MSD USA. No competing interests declared by other authors.

\section{References}

1. Lauro A, Pagano N, Impellizzeri G, Cervellera M, Tonini V. Emergency endoscopy during the SARS-CoV-2 pandemic in the North of Italy: experience from St. Orsola University Hospital-Bologna. Dig Dis Sci. 2020;65:1559-1561. https://doi.org/10.1007/s1062 0-020-06270-x.

2. Lui RN, Wong SH, Sánchez-Luna SA, et al. Overview of guidance for endoscopy during the coronavirus disease 2019 (COVID-19) pandemic. J Gastroenterol Hepatol. 2020;35:749-759.

3. Sultan S, Lim JK, Altayar O, et al. AGA institute rapid recommendations for gastrointestinal procedures during the COVID-19 pandemic. Gastroenterology. 2020. https://doi.org/10.1053/j.gastr o.2020.03.072.

4. Sorbello M, El-Boghdadly K, Airway Petrini F. Airway management in coronavirus disease 2019: In the den of the beast. Anesth Analg. 2020. https://doi.org/10.1213/ane.0000000000004883.

5. Sorbello M, El-Boghdadly K, Di Giacinto I, et al. on behalf of The Società Italiana di Anestesia Analgesia Rianimazione e Terapia Intensiva (SIAARTI) Airway Research Group, and The European
Airway Management Society. The Italian coronavirus disease 2019 outbreak: experiences and recommendations from clinical practice. Anaesthesia. 2020;75:724-732. https://doi.org/10.1111/ anae. 15049 .

6. Corral JE, Hoogenboom SA, Kröner PT, et al. COVID-19 polymerase chain reaction testing before endoscopy: an economic analysis. Gastrointest Endosc. 2020. https://doi.org/10.1016/j. gie.2020.04.049.

7. AGA/DHPA joint guidance for resumption of elective endoscopy. Recommendations for resumption of elective endoscopy during the COVID-19 pandemic. https://gastro.org/news/aga-dhpa-joint -guidance-for-resumption-of-elective-endoscopy/. Accessed 10.06.20.

8. Watson J, Whiting PF, Brush JE. Interpreting a covid-19 test result. BMJ. 2020;369:m1808.

9. Luo M, Cao S, Wei L, et al. Precautions for intubating patients with COVID-19. Anesthesiology. 2020;132:1616-1618. https:// doi.org/10.1097/aln.0000000000003288.

10. Vavricka SR, Tutuian R, Imhof A, et al. Air suctioning during colon biopsy forceps removal reduces bacterial air contamination in the endoscopy suite. Endoscopy. 2010;42:736-741.

11. Lyons C, Callaghan $\mathrm{M}$. The use of high-flow nasal oxygen in COVID-19. Anaesthesia. 2020;75:843-847. https://doi. org/10.1111/anae.15073.

12. Thiruvenkatarajan V, Wong DT, Kothandan H, et al. Airway management in the operating room and interventional suites in known or suspected COVID-19 adult patients: a practical review. Anesth Analg. 2020. https://doi.org/10.1213/ane.0000000000005043.

13. Brewster DJ, Chrimes NC, Do TBT, et al. Consensus statement: safe Airway Society principles of airway management and tracheal intubation specific to the COVID-19 adult patient group. Med J Austr. 2020. https://doi.org/10.5694/mja2.50598.

14. The Faculty of Intensive Care Medicine. Use of supraglottic airwyas during the COVID-19 pandemic. ICM Anaesthesia COVID-19. https://icmanaesthesiacovid-19.org/use-of-supraglott ic-airways-during-the-covid-19-pandemic. Published 7 May 2020. Accessed 8.5.20.

15. Terblanche N, Middleton C, Choi-Lundberg D, Skinner MW. Efficacy of a new dual channel laryngeal mask airway, the LMA ${ }^{\circledR}$ Gastro $^{\mathrm{TM}}$ Airway, for upper gastrointestinal endoscopy: a prospective observational study. Br J Anaesth. 2018;120:353-360.

16. Cook TM, El-Boghdadly K, McGuire B, McNarry AF, Patel A, Higgs A. Consensus guidelines for managing the airway in patients with COVID-19: Guidelines from the Difficult Airway Society, the Association of Anaesthetists the Intensive Care Society, the Faculty of Intensive Care Medicine and the Royal College of Anaesthetists. Anaesthesia. 2020;75:785-799.

17. Wong P, Lim WY, Mok M. Supraglottic airway guided intubation during the COVID-19 pandemic: a closed technique. Anesth Analg. 2020. https://doi.org/10.1213/ane.0000000000004951.

18. Tran A, Thiruvenkatarajan V, Wahba M, et al. LMA ${ }^{\circledR}$ Gastro $^{\mathrm{TM}}$ Airway for endoscopic retrograde cholangiopancreatography: a retrospective observational analysis. BMC Anesthesiol. 2020;20:113.

19. Goudra B, Singh PM. Airway management during upper gi endoscopic procedures: state of the art review. Dig Dis Sci. 2017;62:45-53, 553-554

Publisher's Note Springer Nature remains neutral with regard to jurisdictional claims in published maps and institutional affiliations. 